March 2, 2022

\title{
Accretion Disk Model of Short-Timescale Intermittent Activity in Young Radio Sources
}

\author{
Bożena Czerny ${ }^{1}$, Aneta Siemiginowska ${ }^{2}$, Agnieszka Janiuk ${ }^{1}$, Błażej Nikiel-Wroczyński ${ }^{1,3}$, \\ Łukasz Stawarz ${ }^{4,3}$ \\ ${ }^{1}$ Nicolaus Copernicus Astronomical Center, ul. Bartycka 18, 00-716 Warsaw, Poland \\ ${ }^{2}$ Harvard Smithsonian Center for Astrophysics, 60 Garden St, Cambridge, MA 02138, USA \\ ${ }^{3}$ Astronomical Observatory, Jagiellonian University, Orla 171, 30-244 Cracow, Poland \\ ${ }^{4}$ Kavli Institute for Particle Astrophysics and Cosmology, Stanford University, Stanford, \\ CA 94305, USA \\ bcz@camk.edu.pl
}

\begin{abstract}
We associate the existence of short-lived compact radio sources with the intermittent activity of the central engine caused by a radiation pressure instability within an accretion disk. Such objects may constitute a numerous sub-class of Giga-Hertz Peaked Spectrum sources, in accordance with the population studies of radio-loud active galaxies, as well as detailed investigations of their radio morphologies. We perform the model computations assuming the viscosity parametrization as proportional to a geometrical mean of the total and gas pressure. The implied timescales are consistent with the observed ages of the sources. The duration of an active phase for a moderate accretion rate is short enough $\left(<10^{3}-10^{4}\right.$ years $)$ that the ejecta are confined within the host galaxy and thus these sources cannot evolve into large size radio galaxies unless they are close to the Eddington limit.
\end{abstract}

Subject headings: accretion, accretion disks - black hole physics, instabilities galaxies:active, quasars 


\section{Introduction}

The duty cycle of active galactic nuclei is still a subject of debate. Different mechanisms are likely to operate. On long timescales, up to $10^{8} \mathrm{yrs}$, the merger activity is broadly considered as the cause of an enhanced accretion flow and the main provider of material for the black hole growth. However, at later stages, active galactic nuclei are also likely to pass through subsequent stages of higher and lower activity. Intermittent behavior of this type is likely to be caused by minor mergers or instabilities in the accretion flow.

There are several observational arguments for this intermittent behavior. For example, morphology of X-ray clusters studied with Chandra X-ray Observatory and XMM-Newton indicate a past intermittent AGN activity of the central dominant galaxy that is responsible for heating of the central cluster regions. X-ray images show cavities and ripples that give the timescales of repetitive outbursts and provide a measure of their power (e.g. Ruszkowski et al. 2004; McNamara \& Nulsen 2007; Sanders \& Fabian 2008). Such AGN intermittency seems to be closely related to the feedback process operating in galaxy clusters.

Radio galaxies that are not associated with clusters, also provide signatures of the intermittent activity. First, in many cases studies of radio structure directly showed evidence of two or more of active periods (e.g. Burns et al. 1983; Bridle et al. 1989; Clarke et al. 1992; Schoenmakers et al. 2000a,b; Marecki et al. 2003; Jamrozy et al. 2007). In some sources the new radio structure is highly inclined to the old one (e.g. Cheung 2007), thus indicating an independent accretion/black hole merger episode resulting in renewing activity of the central engine accompanied by the re-orientation of the jet axis (e.g. Merritt \& Ekers 2002; Gopal-Krishna et al. 2003; Blundell 2008; Lal et al. 2008). Some sources of this type are very compact, only a few kpc in size (Marecki et al. 2006). In other sources, both structures (old and new) are aligned (e.g. Saripalli et al. 1996; Schoenmakers et al. 2000a,b; Konar et al. 2006; Marecki et al. 2006), which is consistent with the development of perturbations in the accretion rate within the existing accretion disks and subsequent intermittent radio activity along the same (unchanged) direction of the outflow (Siemiginowska et al. 1996; Siemiginowska \& Elvis 1997; Hatziminaoglou et al. 2001; Janiuk et al. 2004). An extreme example of such an activity is a triple-double source in which three periods of activity led to the aligned features (B0925+420; Brocksopp et al. 2007).

Second, the population studies show the existence of far too many compact ("young") sources in comparison with the number of galaxies with extended old radio structures (O'Dea \& Baum 1997; Snellen et al. 2000) although the full discussion is complex due to various limitations of the available samples (Tinti \& De Zotti 2006). If the total activity period lasts $10^{8} \mathrm{yrs}$, the number of sources with the ages below $10^{3}$ yrs should be roughly $10^{5}$ times lower than the number of sources older than $10^{3} \mathrm{yrs}$, which is not the case. One possible explanation 
is that the jet gets frustrated, and the source is in fact old, although it is small in size (van Breugel et al. 1984, Readhead et al. 1996) . However, the important argument against this interpretation is the lack of the required amount of the thermal matter to quench the jet expansion within host galaxies of GPS sources (Siemiginowska et al. 2005, and references therein). A single short-lasting episode of activity can explain the observed ages (Readhead et al. 1996) but there is no natural explanation of such a timescale. Therefore, a possible explanation is that the activity is intermittent and the source is reborn every few thousand years, without displaying any strong evidence of the previous activity phase, as discussed by several authors (e.g. Reynolds \& Begelman 1997; Kaiser et al. 2000; Kunert-Bajraszewska et al. 2005, 2006; Jamrozy et al. 2007; Giroletti 2008). Detection of several candidates for dying compact sources supports this view (e.g. Giroletti et al. 2005; Kunert-Bajraszewska et al. 2006; Parma et al. 2007).

An accretion disk scenario is a likely explanation of the intermittent behavior on timescales much shorter than $10^{8}$ yrs. Studies of accretion disks in other accreting objects show that cold accretion disks are subject to two types of instabilities: ionization instability, operating on longer timescales and responsible for dwarf nova and X-ray nova phenomenon (Osaki 1974; Meyer \& Meyer-Hofmeister 1981; Smak 1982; see Lasota 2001 for a review), and radiation pressure instability, operating on shorter timescales and responsible for regular outbursts in GRS 1915+105 (Nayakshin et al. 2000; Janiuk et al. 2000, 2002; Merloni \& Nayakshin 2006; see also Fender \& Belloni 2004, and Done et al. 2004 for general reviews on this source). The ionization instability in the context of AGN was studied by a number of authors (Lin \& Shields 1986; Clarke 1988; Mineshige \& Shields 1990; Siemiginowska et al. 1996; Siemiginowska \& Elvis 1997; Hatziminaoglou et al. 2001; Janiuk et al. 2004). The derived separations between outbursts were on order of $10^{6}$ yrs for a $10^{8} M_{\odot}$ black hole, while the outburst duration was an order of magnitude shorter.

In this paper we concentrate on the shorter timescale, radiation pressure driven instability operating above the threshold accretion rate $\dot{m}=0.025$ (in the Eddington units), and we test whether this mechanism is likely to be responsible for very short average ages of Compact Symmetric Objects (CSO), a subgroup of GigaHertz Peaked Sources (GPS). In Section 2, we present our sample of GPS sources. In Section 3, we present our model of the time evolution of the accretion disk under the radiation pressure instability, and in Section 4 we show the resulting intermittent activity patterns for a range of black hole mass, accretion rates and viscosity coefficients. In Section 5 we discuss our results. Throughout this paper we use the cosmological parameters based on the WMAP measurements (Spergel et al. 2003): $\mathrm{H}_{0}=71 \mathrm{~km} \mathrm{sec}^{-1} \mathrm{Mpc}^{-1}, \Omega_{M}=0.27$, and $\Omega_{\mathrm{vac}}=0.73$. 


\section{Young Radio Sources: Timescales and Energetics}

Giga-Hertz Peaked Spectrum (GPS) radio sources are compact and selected based on the convex shape of the radio spectrum that peaks at $\mathrm{GHz}$ frequencies (for a review see O'Dea 1998). They have a compact radio morphology with the radio emission contained within $<1$ kpc (O'Dea 1998) of the host galaxy center. Their radio morphologies on miliarcsec scales resemble the ones observed in large $(\gg 10 \mathrm{kpc})$ radio galaxies with lobes, terminal hot spots and, in some cases, clear core-jets structures. Because of this similarity and the observed high radio power the GPS sources are thought to be the precursors of large radio galaxies observed at the early stage of their expansion (Fanti et al 1995; O'Dea \& Baum 1997; O'Dea 1998). However, as noted above, the number of these sources is too high to support a uniform self-consistent expansion from a compact to a large megaparsec scale radio source. In order to explain the observed overabundance of the compact sources, Reynolds \& Begelman (1997) suggested an intermittent activity of radio-loud active galaxies, with the characteristic activity timescales of $10^{4}-10^{5}$ yrs based on the observed statistics (O'Dea \& Baum 1997).

Compact Symmetric Objects (CSO) comprise a subgroup of GPS sources that was introduced as a separate class over 10 years ago (Wilkinson et al. 1994) on the basis of their "classical double" but extremely compact $(<500$ pc) radio morphology. The small size of the radio structures was usually interpreted as an indication of the age below $10^{4}$ yrs (Readhead et al. 1996; Owsianik \& Conway 1998; Owsianik, Conway \& Polatidis 1998; Taylor et al. 2000; for an alternative suggestion of a frustrated jet see Carvalho 1994, 1998). The strong observational support for the source's young age comes from VLBI monitoring observations and measured hot spots propagation velocities $\sim 0.1-0.2 c$ indicating the kinematic ages of $\sim 10^{3}$ yrs (Conway 2002; Polatidis \& Conway 2003). It should be noted that there is no debate on the intrinsic compactness of CSOs, as opposed to many GPS objects displaying a clear core-jet morphology, since the latter ones may appear rather small due to the projection effects.

We compiled a sample of the GPS/CSO sources from the data available in the literature. In this sample, 10 sources with a measured age are from Polatidis \& Conway (2003) and 14 sources come from the studies of the COINS sample by Gugliucci et al. (2005, 2007). The kinematic ages of these sources were determined on the basis of the measured hot spot separation speed. 47 sources are taken from a much larger sample of GPS sources (including larger size, 1 - $10 \mathrm{kpc}$ Compact Steep Spectrum, CSS) that were studied by Murgia et al. (1999). Determination of their ages is based on the synchrotron cooling time that gives a rather high upper limit. These sources had a significant dispersion of the physical dimensions and derived ages. Note that Murgia et al. (1999) distinguished two classes of sources: the 


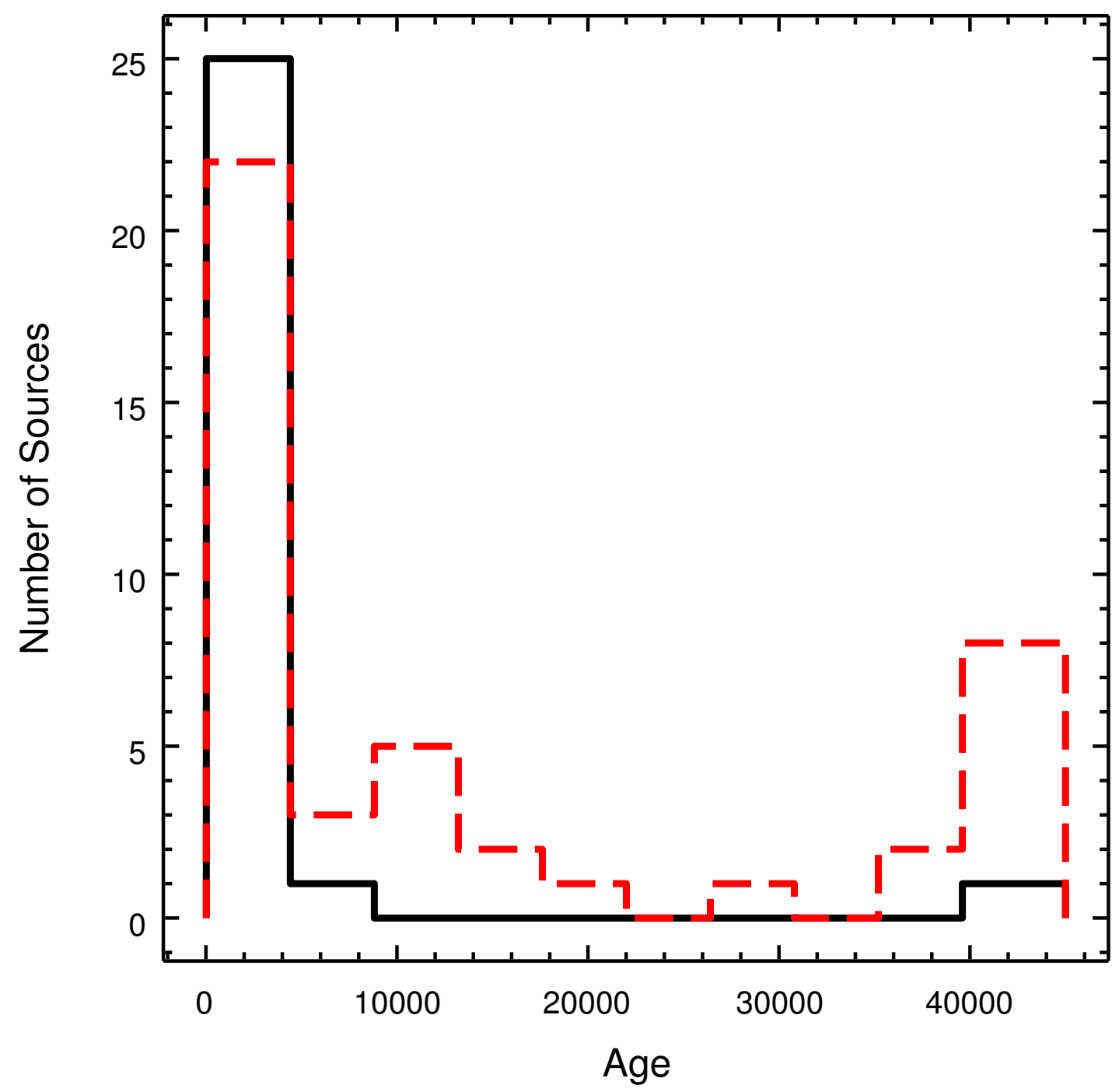

Fig. 1. - The age distribution of the sources in our sample. The black solid line shows the distribution of the sources with the age measured by the kinematic method and the red dashed line is the distribution of the ages measured by the synchrotron method. 


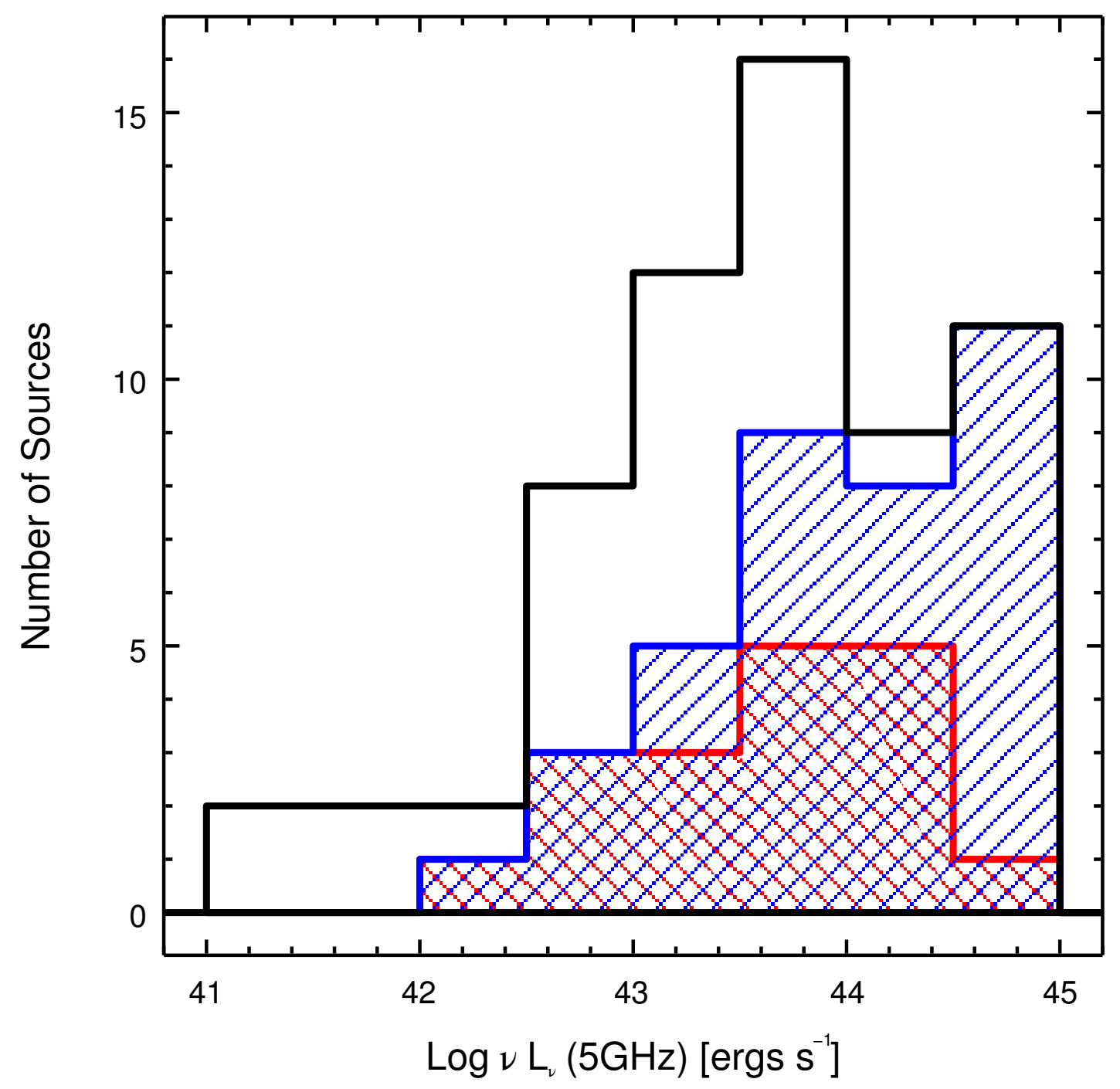

Fig. 2.- The distribution of the $5 \mathrm{GHz}$ radio luminosity for the 72 sources in our sample (black continuous line, whole histogram). We also show the contribution of different subclasses according to the source type: type a (cross-shaded area), type b (shaded area) and the remaining sources (unshaded area). For source classification see Table 1). 
radio spectrum dominated by (a) lobes or (b) jets and hot spots. The sources within the class "a" have the morphology similar to the CSO sources and exhibit a correlation between the source age and the radio linear size. Three individual sources were taken from other publications (Orienti et al. 2007; Tschager et al. 2000).

The whole sample consists of 72 objects with a measured age, and the details are provided in Table 1. The majority of the age values are between $200 \mathrm{yrs}$ and $10^{4} \mathrm{yrs}$, with three sources with the spectral ages considerably longer than $10^{5}$ yrs. We plot the age distribution in Fig. 1. The distribution looks strikingly bimodal but this may well be an artifact of the sample composition. Some age values (usually the shorter ones) are based on the kinematic determination and thus represent a lower limit to the source age, while the other age values (usually the longer ones) come from the synchrotron aging method and give an upper limit to the true age of the source (note that effects of radio source expansion, or deviation from the equipartition may result in the true age of a source to be shorter than its synchrotron age estimated for the fixed radiative cooling rate). A larger sample of sources with the ages determined with a single method is necessary to test if any bimodality is really present in the age distribution. On the other hand, we can be reasonably convinced that the ages of the sources in our sample capture the typical age values well.

We estimated the monochromatic radio luminosities $\left(\nu L_{\nu}\right.$ at $\left.5 \mathrm{GHz}\right)$ for all the sources in our sample (see Table 1) using the measured $5 \mathrm{GHz}$ flux density available in the NED1 database. Figure 2 shows the radio luminosity distribution within the sample. These luminosities can be used to infer the bolometric luminosities of the underlying accretion disks. Note first that, assuming the ratio $L_{\mathrm{syn}} / L_{5 \mathrm{GHz}}$ of the order of one and no relativistic beaming (Wilkinson et al. 1994), almost all the collected objects turn out to be characterized by relatively large intrinsic synchrotron powers in a range $L_{\mathrm{syn}} \sim 10^{43}-10^{46} \mathrm{erg} \mathrm{s}^{-1}$. This, with the $10 \%$ radiative efficiency found/claimed in the evolutionary models for young radio sources (De Young 1993, Stawarz et al. 2008), implies the jet kinetic luminosities in the range $L_{\mathrm{j}} \sim 10^{44}-10^{47} \mathrm{erg} \mathrm{s}^{-1}$, as required if GPS sources are indeed precursors of the extended FR I and FR II radio galaxies. Therefore, the estimated jet powers set lower limits on the accretion rates in the considered objects which, with the standard $10 \%$ radiative efficiency of the disk, would correspond to the accretion luminosities $L_{\mathrm{acc}}>L_{5 \mathrm{GHz}}$.

On the other hand, if the observed radio flux is beamed, the intrinsic synchrotron power of the analyzed sources may be smaller than quoted above. However, relativistic beaming is not expected to play any role in the case of CSO objects (believed to be highly inclined), as well as GPS sources with radio fluxes dominated by lobes (class "a"). Only if the contribution

\footnotetext{
${ }^{1}$ http://nedwww.ipac.caltech.edu/index.html
} 
of the inner jets (and possibly hotspots) to the observed radio emission is significant, could the assumed isotropy of the radio flux be questioned. And indeed, the median $5 \mathrm{GHz}$ luminosity of class "b" sources in our sample, $3 \times 10^{44} \mathrm{erg} \mathrm{s}^{-1}$, is one order of magnitude larger than for the corresponding median for rest of the analyzed objects (see Table 1). Such a difference is also apparent in Figure 2, where in addition to the whole sample, radio luminosity distribution is plotted for the two different classes separately. Having said that, majority of the objects included in our sample are of the CSO type or class "a", and thus our averaged estimates presented in this section should be robust.

Meanwhile, five GPS/CSO sources studied with the XMM-Newton showed $2-10 \mathrm{keV}$ luminosities comparable to $\nu L_{\nu}$ measured at $5 \mathrm{GHz}$ (Vink et al. 2006). If the observed Xray fluxes originate within the accretion flow/disk corona, as advocated by several authors (e.g., Guainazzi et al. 2006, and references therein), the bolometric disk luminosities are then expected to be larger than the observed X-ray ones by at least one order of magnitude (Koratkar \& Blaes 1999, Elvis et al. 1994). We further note that the GPS/CSS sample of objects studied with Chandra revealed $2-10 \mathrm{keV}$ fluxes by a factor of $\sim 5$ higher (on average) than $\nu L_{\nu}$ at $5 \mathrm{GHz}$ (Siemiginowska et al. 2008), although the constructed broadband spectral energy distributions showed also a large dispersion2 2 . Thus, the bolometric disk luminosities of GPS sources, including UV part, can easily be as large as $\sim 100 \times L_{5} \mathrm{GHz}$. The caution here is that the observed X-ray fluxes may be dominated by the inverse-Compton emission generated within compact lobes rather than by the accretion flow (Stawarz et al. 2008). Assuming an order of magnitude correcting factor of 100, as a safe and conservative guess, implies the bolometric disk luminosities of the studied young radio sources $L_{\text {acc }} \sim$ $10^{44}-10^{47} \mathrm{erg} \mathrm{s}^{-1}$. If this correction term is much higher some of the sources may slightly exceed the Eddington luminosity, but this may be indeed acceptable for the brightest ones (see e.g. Collin et al. 2002).

Needless to say, the direct detection of the UV disk radiation in GPS/CSO objects is not possible, since the accretion-related emission at photon energies below $1 \mathrm{keV}$ is known to be significantly obscured. Note in this context that measurements of the HI absorption in radio (Morganti et al. 2007, Pihlström et al. 2003) and the total absorption in X-rays (Guainazzi et al. 2006, Vink et al 2006, Siemiginowska et al. 2008, Tengstrand et al 2008) show significant hydrogen column densities $\left(N_{H} \sim 10^{22} \mathrm{~cm}^{-2}\right)$ in all the studied GPS sources. In addition, the other independent estimates of the bolometric luminosities on the basis of the ionization power do not seem to work in young radio galaxies, as all the analyzed GPS objects are underluminous in [OIII] line, as if the Narrow Line Region is still in the formation

\footnotetext{
${ }^{2}$ Note in this context that in some radio-loud quasars the $2-10 \mathrm{keV}$ luminosities are higher than the monochromatic $\nu L_{\nu}$ at $5 \mathrm{GHz}$ flux by a factor of $\sim 300$ (Tengstrand et al 2008).
} 
process (Vink et al. 2006), being in addition possibly modified by the interaction with the expanding radio lobes (Labiano 2008).

Only a few sources have the measurements of a black hole mass and we give them in Table 2. The mass estimates are based on properties of emission lines and the correlation established by the reverberation mapping (Vestergaard \& Peterson 2006). These few measurements suggest that the black holes in the sources of our sample cover a mass range from $M \sim 10^{8} M_{\odot}$ to $M \sim 3 \times 10^{9} M_{\odot}$, slightly higher than typical masses of radio sources in the Snellen et al. $(2003)$ sample $\left(\sim 10^{8} M_{\odot}\right)$. Also, for a given black hole mass, the younger sources seem to have higher radio luminosities. However, based on this small number of sources we cannot formally assess any trends between the age and the radio luminosity. Unfortunately, without black hole mass measurements for majority of the studied objects, and with only roughly estimated bolometric disk luminosities, we cannot directly calculate the corresponding Eddington ratios. However, if we assume that the representative value of the black hole mass in our sample is the median from Table 2 , i.e. $M \sim 3 \times 10^{8} M_{\odot}$, and take the median monochromatic radio luminosity (for CSOs and class "a" sources only) from Table 1, namely $L_{5 \mathrm{GHz}} \sim 3 \times 10^{43} \mathrm{erg} \mathrm{s}^{-1}$, then with the anticipated correction factor of 100 one obtains the Eddington ratio on the order of $\dot{m} \sim 0.1$.

In addition to such average constraints, for those few sources with the available masses of black holes we can estimate more precisely the bolometric luminosities and the Eddington ratios, assuming the observed fluxes given by Netzer et al (1996) and the template AGN spectrum given by Elvis et al. (1994). The resulting luminosities and accretion rate values

are given in Table 2, and these agree with the rough average estimates given above. Note that, most importantly, the calculated accretion rates exceed the threshold for the radiation pressure instability, $(\dot{m}=0.025)$, for each analyzed source. The bolometric corrections to 5 $\mathrm{GHz}$ flux for those sources are also given in Table 2, and they range from 41 up to 980.

\section{The Model}

We model the time evolution of an accretion disk under the radiation pressure instability. We adopt the viscosity law using the geometrical mean between the gas and the total pressure for the scaling of the stress, i.e. $T_{\mathrm{r} \phi} \propto \alpha\left(P_{\text {gas }} P_{\text {tot }}\right)^{1 / 2}$. As was shown by Merloni \& Nayakshin (2006), such a viscosity law nicely reproduces the effective viscosity resulting from the magnetorotational instability, and describes well the behaviour of the Galactic sources. This viscosity law is also favored for the black hole X-ray binaries (Done \& Davis 2008).

We are using the time-dependent numerical code developed by ourselves and already 
presented in a number of works (Janiuk, Czerny \& Siemiginowska 2002; Janiuk \& Czerny 2005; Czerny et al. 2008). The initial conditions are determined by solving the energy balance, $F_{\text {tot }}=Q_{\text {visc }}^{+}=Q_{\text {adv }}^{-}+Q_{\text {rad }}^{-}$, at every disk radius $r$. Here, the total energy flux $F_{\text {tot }}$ dissipated within the disk at a radius $r$ is calculated as:

$$
F_{\text {tot }}=\frac{3 G M \dot{M}}{8 \pi r^{3}} f(r)
$$

where the boundary condition is $f(r)=1-\sqrt{\frac{3}{r}}$. The cooling and heating terms in this vertically integrated (over the scale $H$ ) energy balance are:

$$
Q_{\mathrm{visc}}^{+}=\frac{3}{2} \alpha \Omega H \sqrt{P_{\text {gas }} P_{\text {tot }}}
$$

where $\alpha$ is the viscosity parameter,

$$
Q_{\mathrm{rad}}^{-}=\frac{P_{\mathrm{rad}} c}{\tau}=\frac{\sigma T^{4}}{\kappa \Sigma}
$$

and

$$
Q_{\mathrm{adv}}^{-}=\frac{2 r P q_{\mathrm{adv}}}{3 \rho G M} F_{\mathrm{tot}}
$$

with $q_{\text {adv }} \approx$ const $\sim 1.0$. The gas and radiation pressure are given by $P_{\text {gas }}=k_{\mathrm{B}} / m_{\mathrm{p}} \rho T$ and $P_{\text {rad }}=4 / 3 \sigma T^{4}, \tau$ is the optical depth, $\Sigma=\rho H$ is the gas column density, $c, k_{\mathrm{B}}$ and $\sigma$ are physical constants, and we adopt the electron scattering opacity $\kappa=0.34 \mathrm{~cm}^{2} \mathrm{~g}^{-1}$. The hydrostatic balance equation gives $\frac{P}{\rho}=\Omega^{2} H^{2}$, and the Keplerian angular velocity $\Omega$ is assumed.

We solve the basic evolutionary equations in 1-D and compute the thermal and viscous evolution of the accretion disk:

$$
\frac{\partial \Sigma}{\partial t}=\frac{1}{r} \frac{\partial}{\partial r}\left(3 r^{1 / 2} \frac{\partial}{\partial r}\left(r^{1 / 2} \nu \Sigma\right)\right)
$$

and

$$
\begin{aligned}
\frac{\partial T}{\partial t}+v_{\mathrm{r}} \frac{\partial T}{\partial r} & =\frac{T}{\Sigma} \frac{4-3 \beta}{12-10.5 \beta}\left(\frac{\partial \Sigma}{\partial t}+v_{\mathrm{r}} \frac{\partial \Sigma}{\partial r}\right) \\
& +\frac{T}{P H} \frac{1}{12-10.5 \beta}\left(Q^{+}-Q^{-}\right) .
\end{aligned}
$$

Here

$$
v_{\mathrm{r}}=\frac{3}{\Sigma r^{1 / 2}} \frac{\partial}{\partial r}\left(\nu \Sigma r^{1 / 2}\right)
$$

is the radial velocity in the disk while $\nu=\left(2 \sqrt{P_{\text {gas }} P_{\text {tot }}} \alpha\right) /(3 \rho \Omega)$ is the kinematic viscosity and $\beta$ is the ratio of the gas pressure to the total pressure, $\beta=P_{\text {gas }} / P$. 
The code also incorporates the option of a two-phase accretion, with the mass exchange between the disk and a hot corona, which allows us for performing quasi-2D computations. It can also include the effect of the jet formation in parametric description. However, in the present work, we concentrate only on the evolution of the disk, so the corona and jet are neglected in order to keep the picture as simple as possible. The models are thus parameterized by the black hole mass, $M$, dimensionless accretion rate, $\dot{m}$, measured in the Eddington units $\left(\dot{M}_{E d d}=3.53\left(M / 10^{8} M_{\odot}\right) M_{\odot} \mathrm{yr}^{-1}\right)$ and the viscosity parameter $\alpha$.

\section{Results}

The time evolution of an accretion disk under the radiation pressure instability is rather fast, in contrast with expectations based on the partial ionization instability. The exemplary lightcurves representing the evolution of the disk bolometric luminosity for a black hole of mass $M=10^{8} M_{\odot}$ are shown in Fig. 3. We performed computations for a range of black hole masses likely to be appropriate for GPS sources $\left(M=10^{7}-3 \times 10^{9} M_{\odot}\right)$. The expected durations of outbursts are shown in Fig. 4 and Fig. 5 for the value of the viscosity parameter $\alpha$ equal 0.2 and 0.02 , correspondingly. The duration of an outburst depends almost linearly on the black hole mass, and strongly varies with the accretion rate. For sources close to the Eddington limit expected outbursts last $10^{4}-10^{5}$ yrs for the smaller value of the viscosity parameter (0.02) and about ten times shorter for the larger viscosity (0.2). Still shorter outbursts are expected for sub-Eddington rates.

The overall range of the observed source ages $\left(200-3 \times 10^{5} \mathrm{yrs}\right)$ is roughly consistent with the expected outburst duration for the value of viscosity parameter $\alpha=0.02$. The larger viscosity parameter $(\alpha=0.2)$ requires super-Eddington accretion rates to explain the duration of the oldest sources ( $>10^{5}$ years). Since we do not have the exact black hole mass determinations for the sources in our sample (Table1) we cannot directly locate them on the the contour maps shown in Figs. 4 and 5 . However, the lines of a constant time duration of an outburst are almost parallel to the lines of a constant bolometric luminosity for a source with an unknown black hole mass. Therefore, for a given estimate of the source bolometric luminosity we predict almost uniquely the duration of the outburst. If in addition we relate the bolometric luminosity with the $5 \mathrm{GHz}$ monochromatic luminosity through a bolometric correction $K_{5 G H z}$, we obtain a relation:

$$
\log \left(\frac{T_{\text {burst }}}{\mathrm{yr}}\right) \approx 1.25 \log \left(\frac{\nu L_{\nu}(5 G H z)}{\operatorname{erg} / \mathrm{s}}\right)+0.38 \log \left(\frac{\alpha}{0.02}\right)+1.25 \log K_{5 G H z}-53.6 .
$$

The real age of the source cannot violate this limit, i.e. should be shorter, and on average should correspond to half of the outburst duration with some dispersion around this value. 

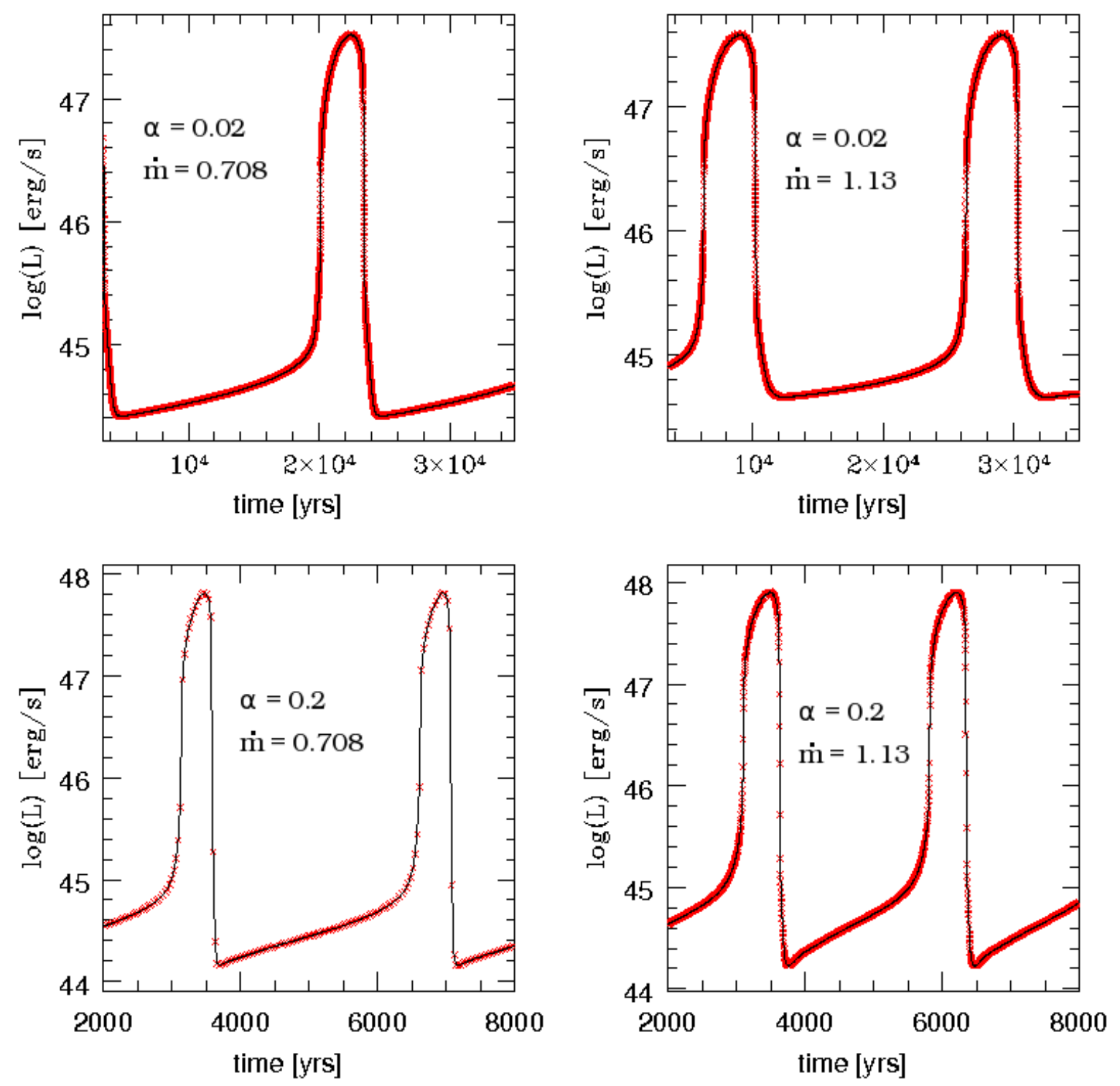

Fig. 3.- Exemplary evolution of the disk bolometric luminosity under the radiation pressure instability $\left(T_{r \phi} \propto \alpha\left(P_{\text {gas }} P_{\text {tot }}\right)^{1 / 2}\right)$ for two exemplary values of the accretion rate $\dot{m}$ (in the Eddington units), and different viscosity parameter $\alpha$. Black hole mass is taken as $M=$ $10^{8} M_{\odot}$. 


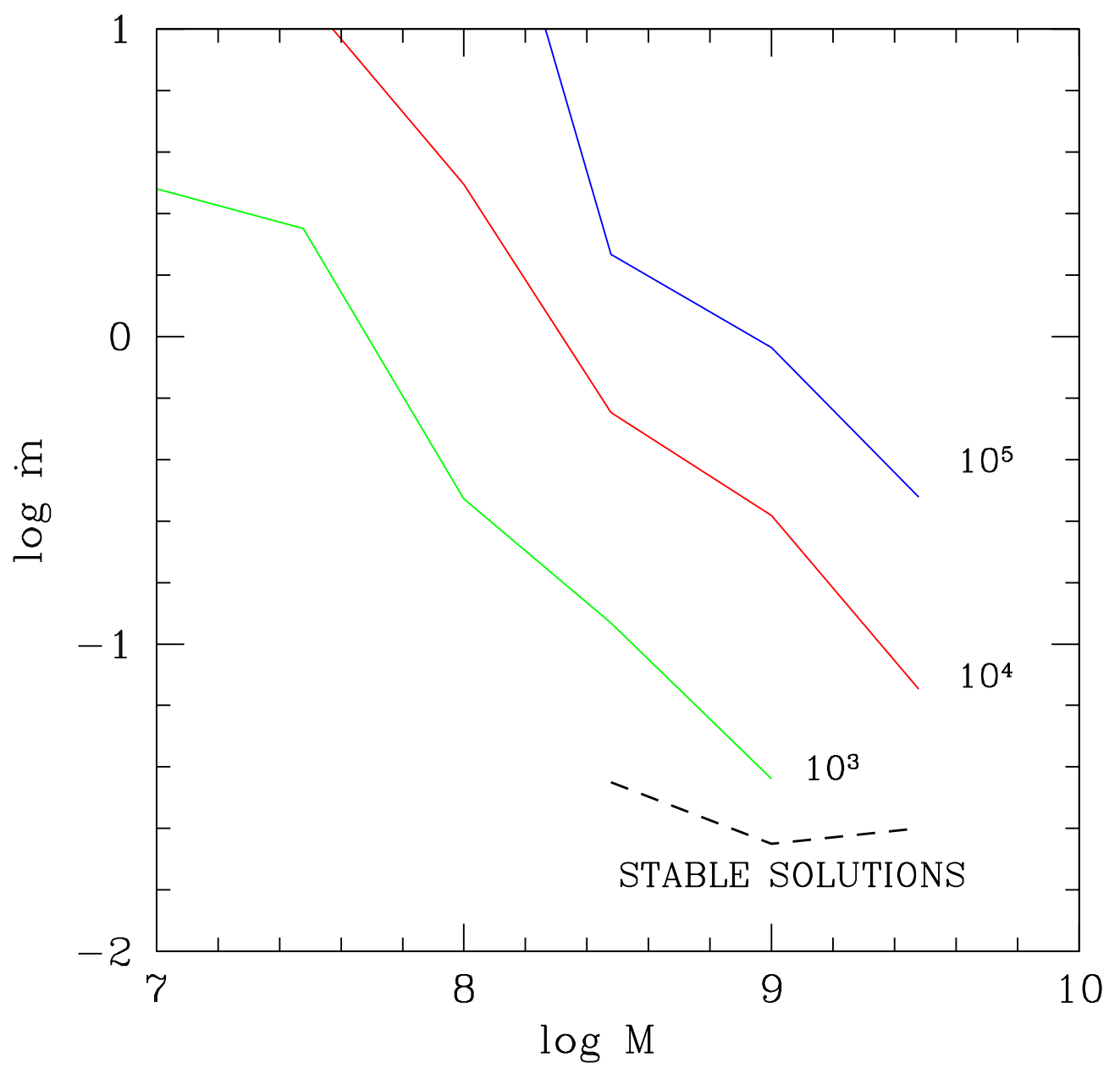

Fig. 4.- Contour maps of the constant outburst duration time, in the black hole mass vs. accretion rate (Eddington units) plane. The outburst durations are given for each curve in years. The viscosity parameter is taken as $\alpha=0.02$. 


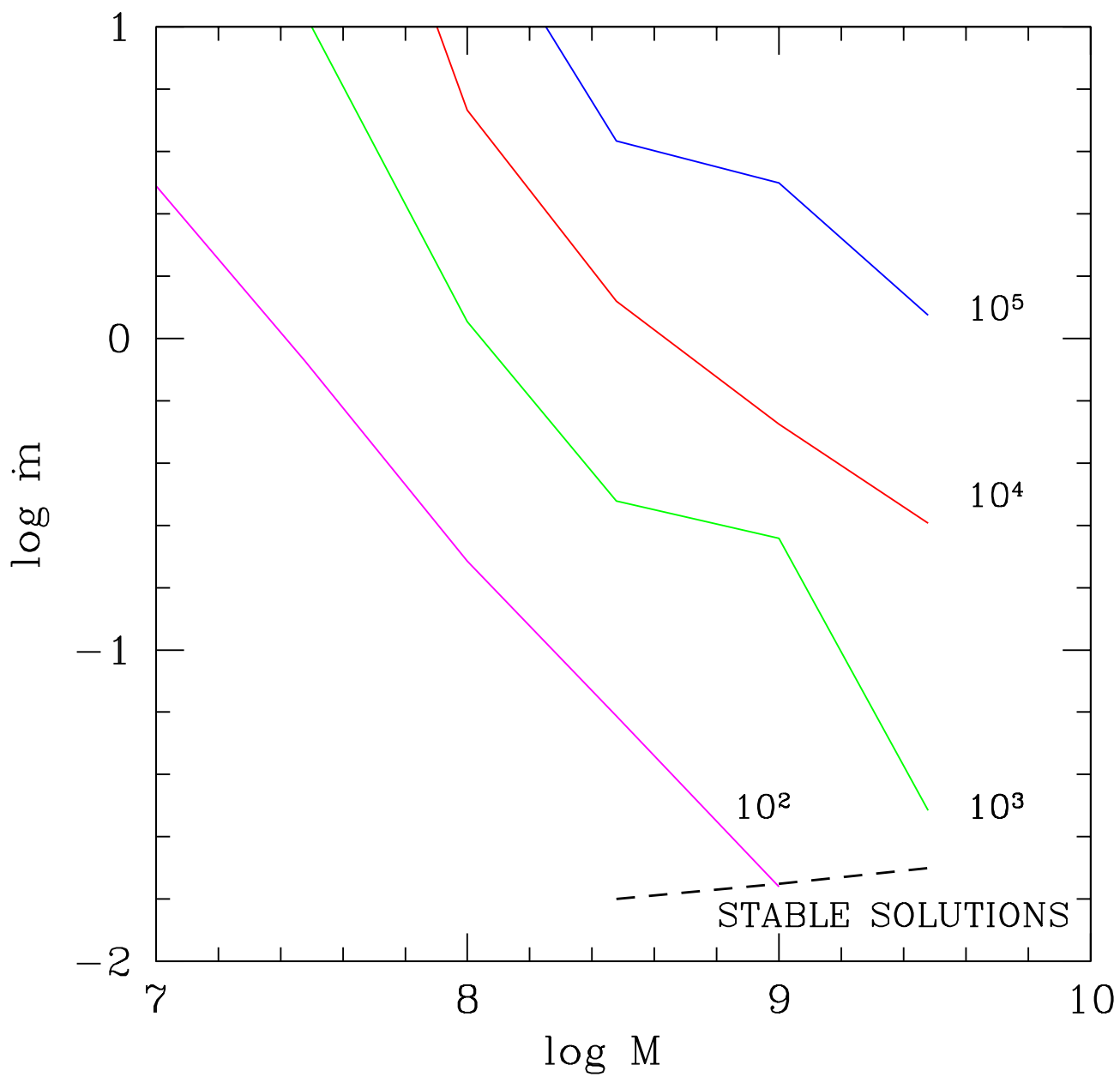

Fig. 5.- Contour maps of the constant outburst duration time, in the black hole mass vs. accretion rate (in Eddington units) plane. The outburst durations are given for each curve in years. The viscosity parameter is taken as $\alpha=0.2$. 


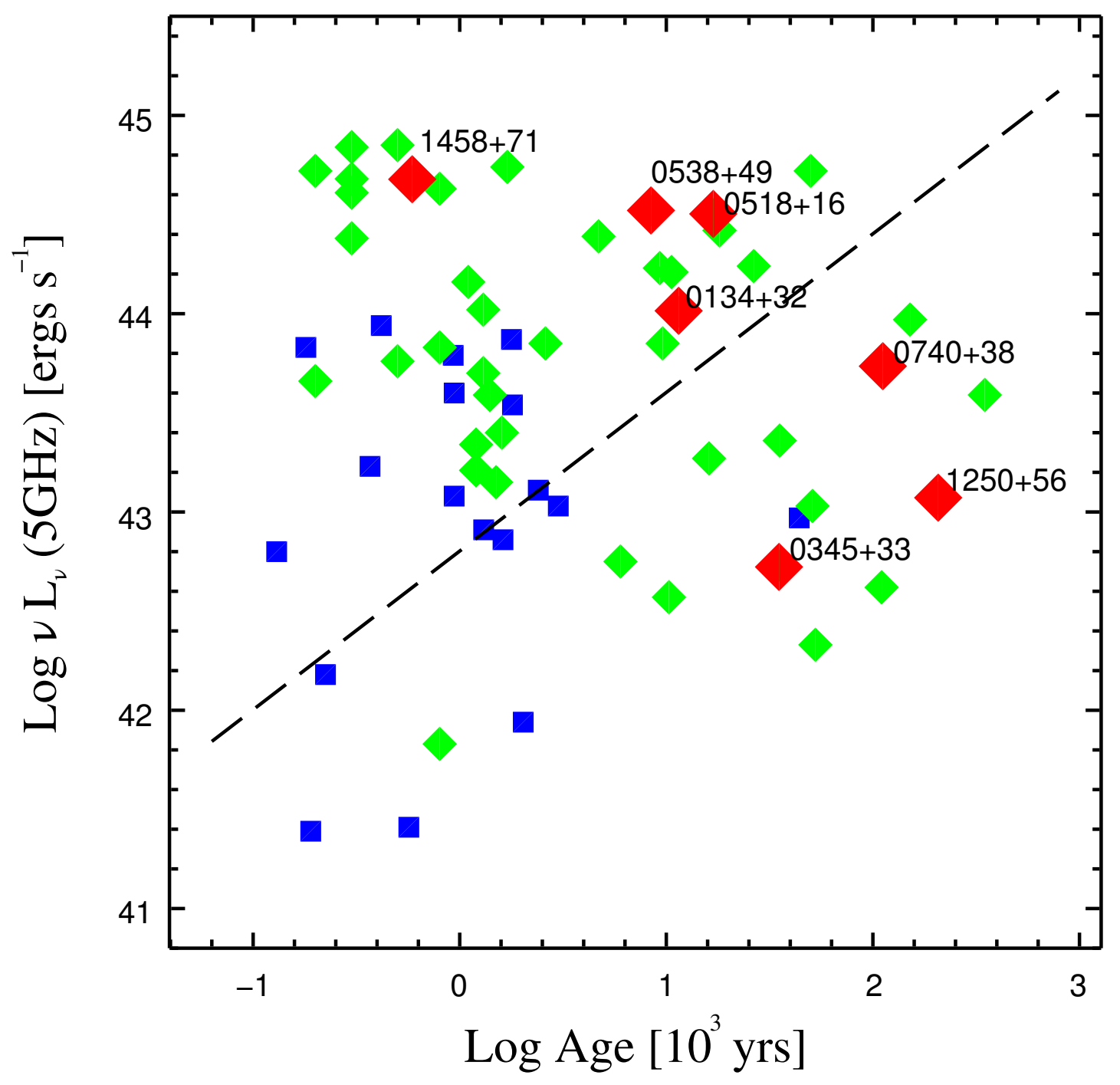

Fig. 6.- Age vs. luminosity diagram for all the sources in the sample. The sources with measured black hole masses are shown by red large diamonds. For those the younger sources show higher luminosity for the same range of mass. More mass measurements are needed to test the radio luminosity vs. age trends. Other sources with synchrotron age determination are shown with green smaller diamonds, and sources with kinematic age determination are shown with blue squares. The dashed lines indicate the duration of an outburst (see Eq.8) for $\alpha=0.02$ and the anticipated bolometric correction factor 300 . 
In Fig. 6, we plot the lines corresponding to Eq. 8 with $\alpha=0.02$ and the anticipated bolometric correction factor 300 (see Sect. 2). On average, most of the sources are located to the left of the line, as expected if the age of the radio structure reflects directly the age of the central engine. However, there are several outliers. Most of them are relatively old, having their ages determined from the synchrotron method (e.g., 1233+418). This method gives an upper limit to the age, and thus the lifetimes of these, being about $\sim 10^{5}$ yrs, may be considerably overestimated. However, four sources with kinematically measured age also show considerable departure to the right from the line. The distance from the predicted line is the largest for $0035+227$. Its radio spectrum is steep (e.g. Anton et al. 2004). Perhaps the luminosity of the source is already dropping down due to aging and therefore our standard relation between the accretion rate in the model and the observed luminosity does not apply.

Also, the sources should not be observed in the range where instability does not operate. The exact boundary of the unstable region is complicated and depends on the accretion rate. We can say that the instability operates if $\dot{m}>0.025$, approximately. None of the sources in our sample with a measured mass violate this limit. As argued in Section 2, we expect that this criterium is also fulfilled on average, at least for the majority of the GPS population.

In Fig. 7 and Fig. 8 we give the outburst separations for lower and higher values of the viscosity coefficient, respectively. As the figure shows, the separation between the outbursts is a complex non-monotonic function of the accretion rate. For high accretion rates, the outburst separation decreases with the accretion rate and increases with the black hole mass. This trend is seen in Figs. 7 and 8 as the upper branches of the constant time separation lines. This part of the plot is similar to the plots of the outburst duration (Figs. 4 and 5), with the outburst separation being typically by a factor of 10 higher. The typical timescales are then consistent with the ones postulated by Reynolds \& Begelman (1997). These timescales give the limits for statistical studies of the galactic activity, and we devote a part of the Discussion to this issue (see Sect. 5.3).

However, when the accretion rate approaches the value for which the instability ceases to exist, the trend with the accretion rate is reversed and the time separation goes to infinity. The limiting value of the accretion rate is specific for the given black hole mass. This is seen as the lower branches of the constant separation time lines. The parameter range (accretion rate and black hole mass) where this happens is relatively narrow. At still lower accretion rates, for a given mass, the disk is stable and there are no outbursts. Such a source should evolve in a continuous rather than intermittent way. 


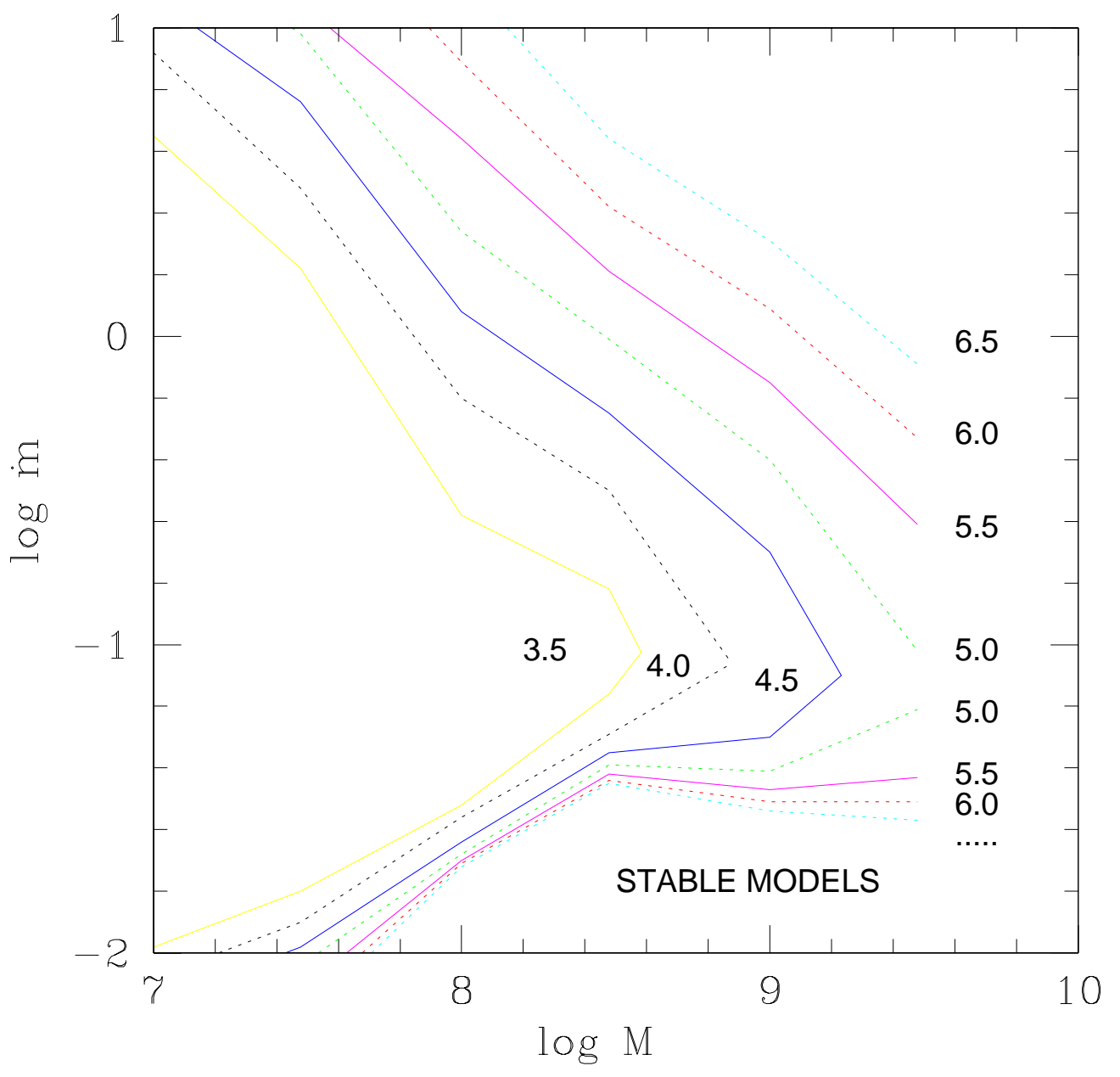

Fig. 7.- Contour maps of the constant outburst separation time, in the black hole mass vs. accretion rate (in the Eddington units). The outburst durations are given for each curve, in $\log (T)$ [yrs]. The viscosity parameter is $\alpha=0.02$. A region of the parameter space below the lowest curve shows shows stable model for accretion rates below the threshold. 


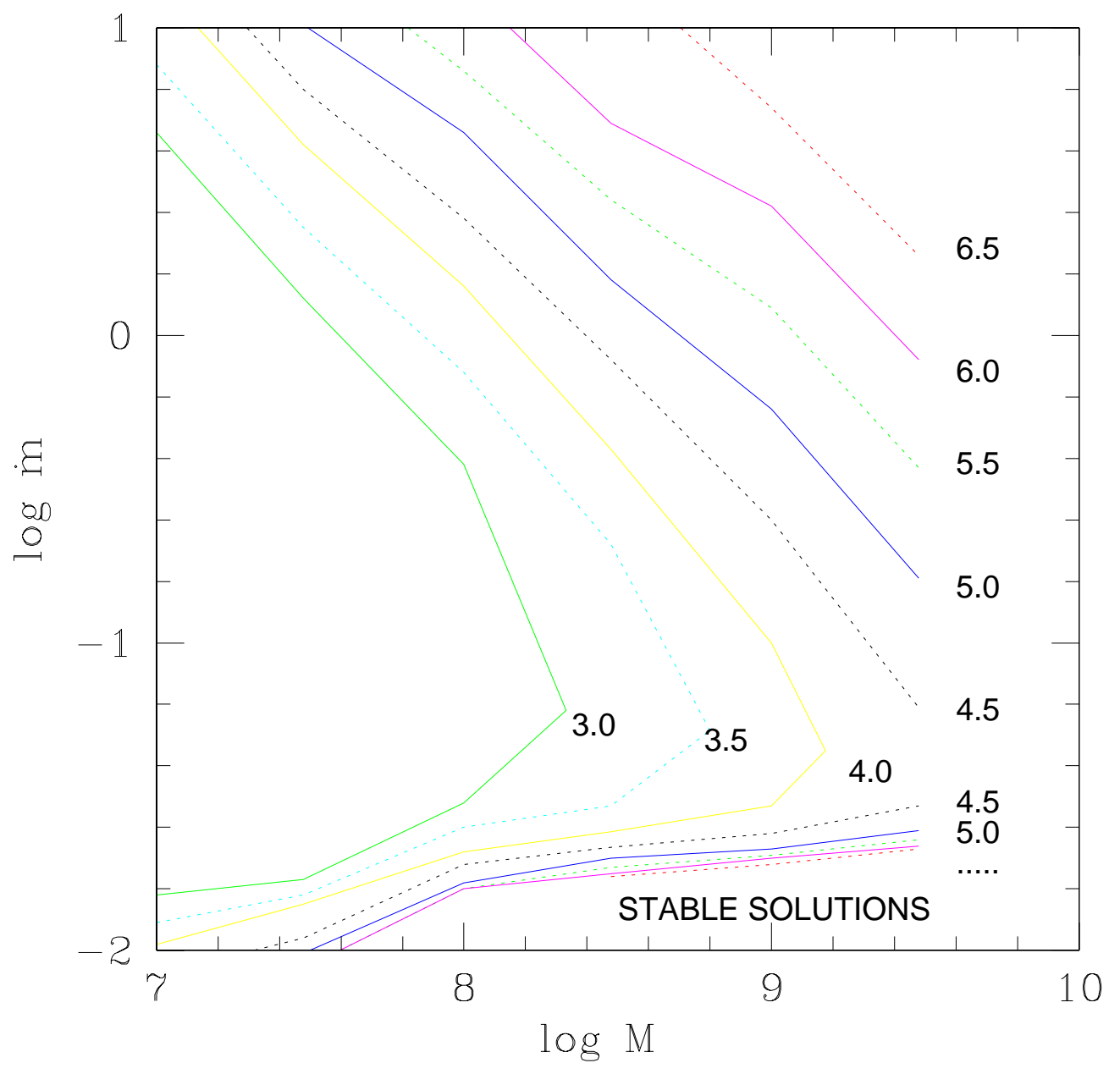

Fig. 8.- Contour maps of the constant outburst separation time, in the black hole mass vs. accretion rate (in the Eddington units). The outburst durations are given for each curve, in $\log (T)$ [yrs]. The viscosity parameter is $\alpha=0.2$. A region of the parameter space below the lowest curve shows shows stable models for accretion rates below the threshold. 


\section{Discussion}

We study the possibility that the observed short lifetimes of (some of) GPS sources reflect the intermittent activity of the nucleus caused by the radiation pressure instability in the accretion disk. We model the disk outbursts using $T_{r \phi} \propto \alpha\left(P_{\text {gas }} P_{\text {tot }}\right)^{1 / 2}$ viscosity law, as previously done by Merloni \& Nayakshin (2006) in the context of Galactic sources. This parametric approach seems to be quite successful. For the smaller of the two values of the considered viscosity parameter $(\alpha=0.02)$, supported by the previous studies of the AGN variability (Siemiginowska \& Czerny 1989; Starling et al. 2004), the theoretically obtained outburst durations for a range of black hole masses and accretion rates are comparable to the ages of the radio structures determined for our sample of 72 GPS/CSO objects. Therefore, the radiation pressure instability mechanism offers an interesting possibility for the intermittency of young radio sources, as postulated by Reynolds \& Begelman (1997) to explain the apparent overabundance of such. The time separations between outbursts obtained from our model are also comparable to those requested by Reynolds \& Begelman. However, our approach leaves several open questions/problems which should be addressed in the future in order to establish the theoretical basis for the intermittent character of jet activity. We discuss these problems below.

\subsection{Radiation pressure instability}

On the theoretical side, it is still unclear whether the radiation pressure instability operates in accretion disks. If the disk viscosity is parameterized as in the classical paper of Shakura \& Sunyaev (1973), the domination by radiation pressure leads to the thermal (Pringle et al. 1973) and viscous (Lightman \& Eardley 1974) instability when the ratio of the gas pressure to the total pressure drops below 0.4 (Shakura \& Sunyaev 1976). If the viscosity scales with the gas pressure, a stable solution for the disk structure is obtained (Sakimoto \& Coroniti 1981), and any intermediate scaling limits the parameter range for the instability (with the scaling adopted in the present paper, this limit is the gas pressure to the total pressure ratio of 3/11; see Szuszkiewicz 1990). However, the viscosity mechanism that operates in the accretion disks is connected with the magnetorotational instability (Balbus \& Hawley 1991). Numerical simulations roughly support some scaling of the effective torque with the pressure (linear for the gas pressure dominated models, e.g. Hawley \& Krolik 2001, and with $P_{\text {rad }}^{0.7}$ scaling for the radiation pressure dominated models, Hirose et al. 2008), although the issue of the global disk stability is unclear. Older hydrodynamical simulations (e.g. Agol et al. 2001) suggested that radiation pressure dominated disks are unstable while in the most recent MHD simulations only a moderate variability was found, without the 
global coherent outbursts (Turner 2004, Hirose et al. 2008). However, these simulations still do not fully describe the accretion process as they are limited to the shearing box approach.

Therefore, at this moment our parametric approach adopted to compare the model predictions with the data seems to be the optimal choice. In case of the regular periodic outbursts of the galactic source GRS 1915+105, lasting from $\sim 100$ to $\sim 2000$ seconds (depending on the source mean luminosity), this approach is successful (e.g. Janiuk et al. 2002), particularly if the viscosity is parameterized as $\alpha\left(P_{\text {gas }} P_{\text {tot }}\right)^{1 / 2}$ (Merloni \& Nayakshin 2006). No other quantitative mechanism has been proposed to explain the observed behavior of this object. The frequently quoted evacuation of the inner disk due to a related outburst is rather unlikely (e.g. Czerny 2006), and only the limit cycle mechanism (likely driven by the radiation pressure instability) explains the absence of the direct transitions from the state $\mathrm{C}$ to the state B in this black hole binary (Janiuk et al. 2002), as well as the observed QPO oscillations (Misra et al. 2006). In addition, scaling the observed GRS $1915+105$ timescale with the black hole mass by a factor $10^{8}$ gives the outbursts durations from 300 yrs to 6000 yrs. This gives an additional, model-independent argument that an intermittency in AGN on the timescales of hundreds/thousands of years is quite likely to be of the similar origin indeed.

\subsection{High and low luminosity states of accretion disk}

The radiation pressure instability implies that an accretion disk alternates between the two basic states which are thermally stable: (1) an outburst, i.e. a high accretion rate state; and (2) a low accretion rate state. During an outburst the disk is almost stationary, with a roughly constant accretion rate at all radii, and models developed for the stationary accretion disks apply to the timescales shorter than the viscous timescale of hundreds/thousands of years (e.g. Czerny 2006). A temporary accretion rate in this state is somewhat higher than the Eddington rate, and higher than the external supply rate to the outer parts of the disk. The disk thermal stability during the outburst is caused mainly by the advection of the part of the energy to the black hole. A low state occurs between the outbursts when the accretion rate falls rapidly and the disk becomes significantly non-stationary - the local accretion rate decreases inwards and the mass accumulates in the disk. The thermal stability in this state is caused by a reduction of the radiation pressure. Additionally, as the accretion rate becomes very low the disk may become optically thin and/or replaced by an ADAF flow in its innermost part (Janiuk et al. 2004). This effect was not included in the computations since the issue of the cold disk evaporation and disk/ADAF transition is a complex and open question (for a recent review, see e.g. Narayan \& McClintock 2008). It probably does not 
affect a global evolution although it would strongly influence the radiation spectrum.

In our model we identified the high accretion rate state of the disk with a high energy output of a jet. This is an assumption since the jet/disk connection in Active Galaxies is not well understood. However, a monotonic relation between the radio power of the jet and the optical luminosity of the active nucleus (and thus accretion rate) is suggested by the data for the entire population of radio loud elliptical-hosted active galaxies (see e.g. Sikora et al. 2007 and references therein). So even though a fraction of the available accretion energy used to power a jet may vary between objects with different Eddington ratios, the high accretion rate radio loud sources generate more powerful jets than the low accretion rate ones. That seems to be true regardless of the fact that the Eddington ratio may not be the only parameter controlling the jet production efficiency in AGN. (e.g. Sikora et al. 2007, for the discussion on a role of a black hole spin)

The radiation pressure instability should also operate in radio-quiet sources. An outburst in this case would correspond to a typical state of a bright radio-quiet quasar with a strong outflow while the low state (between the outbursts) could be identified with sources accreting at the Eddington ratio of about a fraction of a per cent. Such low accretion sources are usually found among Low Luminosity AGN. However, it is not easy to confirm that indeed the bright AGN and (a fraction of) LL AGN are stages of the same major strong activity episode. Observations of distant LL AGN are not available, while the space density of bright nearby quasars is very low, and the local LL AGN are most likely starved due to a shortage of the accreting material. The selected radio-quiet samples are strongly biased against the lower Eddington ratio sources, partially due to a possible transition to an inner radiatively inefficient flow in this state, as recently discussed by Hopkins et al.(2009). In other words, the accretion disk intermittency presented in this paper, when applied to radio quiet AGN may be related to the problem of "missing AGN" in the context of the extragalactic X-ray background. In particular, as noted by Hopkins et al. (2009), "missing" AGN needed to produce the observed diffuse emission above $10 \mathrm{keV}$ may not be heavily obscured, but rather the ones accreting at lower rates. It is interesting to speculate that these are in fact the AGN with their disks in the quiescence, so in between the outbursts driven by the radiation pressure instability.

Another manifestation of the radiation pressure instability would be an observation of a fading quasar. However, the transition from the high to low accretion rate state occurs on thermal timescale which is of order of $\sim 350$ years for a $10^{8} M_{\odot}$ mass black hole, and a factor of ten longer for a typical bright quasar hosting a black hole mass of $10^{9} M_{\odot}$. On the other hand a lower mass Narrow Line Seyfert 1 galaxies might be good candidates for monitoring the effects of the radiation pressure instability. Some of them (although most 
likely not all of them; see e.g. Mathur \& Grupe 2005, Nikolajuk et al. 2009) are accreting at high Eddington ratios. Since black hole masses in NLS1 galaxies are typically of order $10^{6}-10^{7} M_{\odot}$ the activity decay timescales should be shorter, so NLS1 objects should fade and transition to a LL AGN state in 4 - 40 years. A outburst duration is by a factor 11 (for the viscosity parameter 0.02) longer than the fading timescale. Therefore, one out of 11 objects in a long monitored sample should change its spectral state from NLS1 to S1 or LL AGN. An example of the NLS1 source disappearance was found by Grupe et al. (2007), although the observational effect in this case may be caused by a temporary obscuration as this particular source seems to be brightening again on the timescales that are too short to be explained by the state transitions (Grupe et al. 2008).

\subsection{Source evolution between the outbursts}

In the radiation pressure instability scenario, the outbursts repeat regularly, every $10^{4}-$ $10^{6}$ years, as long as there is a considerable supply of accreting matter toward a black hole. If there is one-to-one correspondence between the radio structure and the activity epoch of a central engine, a "young" radio source would just mean one more ongoing episode of the nuclear outburst. Meanwhile, the previous phases of the jet activity should then manifest by the presence of fossil radio lobes in addition to the compact (young) ones, if only the timescale for fading of the latter structures is on average longer than the characteristic quiescence timescale between the activity epochs. Also, some number of dying but compact radio objects lacking active nuclei should be expected, depending on the relative durations characterizing the outbursts, the quiescence periods, and aging of dying/fossil radio lobes. We note that about $10 \%$ of GPS objects (though mostly quasars) show evidences for a faint extended radio structure in addition to the compact ones (Stanghellini et al. 1998, 2005). Also, in some cases the traces of complexity in compact radio morphologies are seen (Jeyakumar et al. 2000). On the other hand, an extensive search for the evidence of radio fossils in 374 radio sources with different ages done by Sirothia et al. (2008) did not bring positive results. This may indicate that the remnants of previous active phases are in general rare, at least in the evolved/old objects. However, finding of such remnants requires deep, low-frequency and high dynamic range radio observations, and thus more analysis is needed before drawing the robust conclusions.

But is the timescale for fading of compact radio lobes which stopped to be supplied by the jet longer than the quiescence periods in between of the accretion disk outbursts? If so, a significant number of fossil radio structure should be indeed expected for GPS sources. If not, the traces of the previous activity phase would be erased before the new radio structure 
is born. Note in this context that Reynolds \& Begelman (1997) assumed that the radio source has an initial outburst that lasts for $\sim 10^{4} \mathrm{yrs}$ and then the power source switches off. The system continues its expansion (coasting phase) that is is still pressure driven and the shocked shell remains highly supersonic for the rest of the expansion period until $\sim 10^{6}$ yrs. In their model, the radio emission fades while the dynamical structure remains basically intact. However, if the source turns off at $\sim 10^{3} \mathrm{yrs}$, i.e. earlier than at the time considered by Reynolds \& Begelman (1997), the high density galactic medium should dramatically impact the source evolution quickly after the power is turned off.

A typical advance speed of the CSO components is on the order of $0.1 c$ (Gugliucci et al. 2005), so during the active phase lasting $t \sim 10^{3}$ yrs the ejecta cover the distance of 300 pc. Further pressure driven expansion proceeds until the accumulated jet energy becomes comparable to the energy content of the heated medium. Assuming the jet energy output rate of $L_{\mathrm{j}} \sim 10^{46} \mathrm{erg} \mathrm{s}^{-1}$, in agreement with the constraints presented in Section 2, and the energy content of the heated medium $E=R^{3} \rho v_{\mathrm{s}}^{2}$, with the number density $n=\rho / m_{\mathrm{p}} \sim 0.1 \mathrm{~cm}^{-3}$ and the sound speed $v_{\mathrm{s}}=\left(5 k T / 3 m_{\mathrm{p}}\right)^{1 / 2} \sim 3.7 \times 10^{7} \mathrm{~cm} \mathrm{~s}^{-1}$ as appropriate for the hot $\left(T \sim 10^{7} \mathrm{~K}\right)$ gas in the central parts of giant elliptical, we obtain the maximum size of the perturbed region of $\sim 3 \mathrm{kpc}$ for $E \sim L_{\mathrm{j}} t$. Note that this is consistent with the recent observations indicating a correlation between the intensity of narrow emission lines (e.g. [OIII] $\lambda 5007 \AA$ ) and a linear size of a young radio source (see Labiano 2008). Because the NLR clouds are distributed within a few kpc from the active nucleus, the overpressured expanding radio lobes with comparable maximum sizes are expected to modify the line luminosity by driving shocks into the ISM, as discussed in Labiano (2008). The time needed for the ejecta to reach this distance with the speed of $0.1 c$ is $3 \times 10^{4} \mathrm{yrs}$. Later, the heated cocoon cools down and recollapses (see e.g. Kaiser et al. 2000). The recollapse phase happens with the sound speed of the heated material, and hence it lasts for about $10^{6}-10^{7} \mathrm{yrs}$. After that time no traces of the past activity are expected. This is however much longer than the outbursts separation times given in Figures 7 and 8 for the anticipated black hole masses and accretion rates.

On the other hand, the recollapse timescale may be shorter if the jet power is significantly lower than $L_{\mathrm{j}} \sim 10^{46} \mathrm{erg} \mathrm{s}^{-1}$. Also, high efficiency in radiating out the jet energy in forms of high-energy photons, or non self-similar evolution of radio cocoons, may play an analogous role. For example, in the non self-similar evolutionary model for GPS radio galaxies considered by Stawarz et al. (2008), the sideway expansion of the compact ( 1 kpc) lobes is expected to be lower than the advance speed of the terminal hotspot by a factor of at least 3, and the radiative jet efficiency is expected to exceed $10 \%$ in the early phases of the source evolution. Such effects can possibly result in fact that the time separation between nuclear outbursts may be long enough to erase the traces of the previous radio structure 
before a new one is born, at least in some objects. An interesting question to ask is how those other objects would look like on radio maps. Note in this context that the new-born jet propagating not within the unperturbed interstellar medium of the host elliptical but within the fossil lobes is expected to display a very narrow radio cocoon and very high $(>0.1 c)$ advance velocity, resulting from the ballistic propagation of the jet in a rarefied environment (Kaiser et al. 2000, Stawarz 2004). Such a morphology would be called the "core-jet" one rather than the "classical double". In other words, the source with the quiescence periods shorter than the timescale for the collapse of the fossil kpc-scale lobes would be classified as a core-jet dominated GPS galaxy rather than CSO. It is typically assumed that the core-jet dominated GPSes are in fact intrinsically similar to CSOs but only viewed at smaller inclination. However, the alternative explanation we propose here is that the former ones represent rather the objects with the outburst and quiescence timescales much shorter $\left(<10^{5}\right.$ yrs $)$ than the latter ones.

Accordingly to the discussion presented above, we can expect that a radio source powered by a short-lived outbursts of the central activity is not be able to escape from the host galaxy. So it will be confined within the host as it is observed in GPS and CSO sources. In order for the radio source to grow a large scale radio structure, similar to the ones observed in FRI/FRII radio galaxies, the active phase needs to last longer than, roughly, $10^{4}$ yrs. This gives us a limit on the accretion rates that are required for the formation of the most extended radio sources. Based on Figures 5 and 6 and assuming $10^{4}$ yrs outburst durations, we estimate such a critical accretion rate to be $0.5 \dot{M}_{\text {Edd }}$ for $\alpha=0.2$, and $0.1 \dot{M}_{\text {Edd }}$ for $\alpha=0.02$. Only few such powerful outbursts would be needed to support the growth of a large radio source beyond the host galaxy.

\subsection{Future applications}

The model predicts a complex behaviour of the radio sources depending on the black hole mass and disk accretion rate: continuous jet activity, intermittent compact sources, and intermittent sources with ejecta leaving the host galaxy. Therefore, it will be interesting to consider carefully statistics of the observed number of radio sources, their lifetimes and sizes, in comparison to the predictions of this model. Perhaps, the largest radio objects experience the most powerful and long-lasting outbursts of the activity. For example, the outbursts detected in the Chandra X-ray images of Perseus cluster last for $\sim 10^{6}$ yrs with a separation time of $\sim 10^{7}$ yrs (Fabian et al. 2003; see McNamara \& Nulsen 2007) for other examples of X-ray clusters). New measurements of the ages in CSO objects, needed for such 
comparison, are continuously coming from VLBA3, and the statistics of jet-related X-ray cavities in galaxy clusters is increasing. Future surveys, like the on-going DEVOS (Deep Extragalactic VLBI-Optical Survey; Mosoni et al. 2006) will bring even more objects that can be analyzed in this respect.

\section{Acknowledgments}

We thank Marek Sikora and Brandon Kelly for very helpful discussions. We alse thank the anonymous referee for comments.

This research has made use of the NASA/IPAC Extragalactic Database (NED) which is operated by the Jet Propulsion Laboratory, California Institute of Technology, under contract with the National Aeronautics and Space Administration.

This work was supported in part by grant 1P03D00829 of the Polish State Committee for Scientific Research and the Polish Astroparticle Network 621/E-78/BWSN-0068/2008.

This research is funded in part by NASA contract NAS8-39073. Partial support for this work was provided by the Chandra grants GO2-3148A, GO5-6113X and GO8-9125A-R.

\section{REFERENCES}

Agol, E., Krolik, J.H., Turner, N.J., \& Stone, M., 2001, ApJ, 558, 543

Anton, S., Browne, I.W.A., Marcha, M.J.M., Bondi, M., \& Polatidis, A., 2004, MNRAS, 352,673

Balbus, S.A., \& Hawley, J.F., 1991, ApJ, 376, 214

Blundell, K.M., 2008, in ASP Conference Series, Vol. 386, p. 467

Bridle, A.H., Fomalont, E.B., Byrd, G.G., \& Valtonen, M.J., 1989, AJ, 97, 674

Brocksopp, C., Kaiser, C.R., Schoenmakers, A.P., \& de Bruyn, A.G., 2007, MNRAS, 382, 1019

Burns, J.O., Schwendeman, E., \& White, R.A., 1983, ApJ, 271, 575

\footnotetext{
${ }^{3}$ Two new sources from Nagai et al. (2008), with both kinematic and synchrotron ages of $\sim 2000$ and $\sim 30 \mathrm{yrs}$, are not included in the present paper.
} 
Carvalho, J.C., 1994, A\&A, 292, 392

Carvalho, J.C., 1998, A\&A, 329, 845

Cheung, C. C. 2007, AJ, 133, 2097

Clarke, C.J., 1988, MNRAS, 235, 881

Clarke, D.A., Bridle, A.H., Burns, J.O., Perley, R.A., \& Norman, M.L., 1992, ApJ, 385, 173

Collin, S., et al. 2002, A\&A, 388, 771

Conway, J.E., 2002, New Astronomy Review, 46, 263

Czerny, B., 2006, ASP Conference Series, 360, 265

Czerny, B., Siemiginowska, A., Janiuk, A. \& Gupta, A.C., 2008, MNRAS, 386, 1557

De Young, D.S., 1993, ApJ, 402, 95

Done, C., \& Davis, S.W., 2008, ApJ, 683, 389

Done, C., Wardzinski, M., \& Gierlinski, M., 2004, MNRAS, 349, 393

Elvis, M. et al., 1994, ApJS, 95, 1

Fabian, A.C. et al., 2003, MNRAS, 344, L34

Fanti, C., Fanti, R., Dallacasa, D., Schilizzi, R.T., Spencer, R.E., \& Stanghellini, C., 1995, A\&A, 302, 317

Fender, R., \& Belloni, T., 2004, ARA\&A, 42, 317

Giovannini, G., Giroletti, M., \& Taylor, G.B., 2007, A\&A, 474, 409

Giroletti, M., 2008, ASP Conf. Ser. 386, p. 176

Giroletti, M., Giovannini, G., \& Taylor G.B., 2005, A\&A, 441, 89

Gopal-Krishna, Bierman, P.L., \& Wiita, P.J., 2003, ApJL, 594, 103

Grupe, D., Schady, P., Leighly, K.M., Komossa, S., O’Brien, P.T., \& Nousek, J.A., 2007, AJ, 133, 1988

Grupe, D., Leighly, K.M., Komossa, S., 2008, AJ, 136, 2343 
Guainazzi, M., Siemiginowska, A., Stanghellini, C., Grandi, P., Piconcelli, E., \& Azubike Ugwoke, C., 2006, A\&A, 446, 87

Gugliucci, N.E., Taylor, G.B., Peck, A.B., \& Giroletti, M., 2005, ApJ, 622, 136

Gugliucci, N.E., Taylor, G.B., Peck, A.B., \& Giroletti, M., 2007, ApJ, 661, 78

Hatziminaoglou, E., Siemiginowska, A., \& Elvis M., 2001, ApJ, 547, 90

Hawley, J.F., \& Krolik, J.H., 2001, 548, 348

Hirose, S., Krolik, J.H., \& Blaes, O., 2008, astro-ph/0809.1708

Hopkins, P.F., Hickox, R., Quataert, E., \& Hernquist, L., 2009, astro-ph/0901.2936

Jamrozy, M., Konar, C., Saikia, D.J., Stawarz, Ł., Mack, H.-K., \& Siemiginowska A., 2007, MNRAS, 378, 581

Janiuk, A., \& Czerny, B., 2005, MNRAS, 356, 205

Janiuk, A., Czerny, B., \& Siemiginowska, A., 2000, ApJL, 542, L33

Janiuk, A., Czerny, B., \& Siemiginowska, A., 2002, ApJ, 576, 908

Janiuk, A., Czerny, B., Siemiginowska, A., \& Szczerba, R., 2004, ApJ, 602, 595

Jeyakumar, S., Saikia, D.J., Pramesh, R.A., \& Balasubramian, V., 2000, A\&A, 362, 27

Kaiser, C.R., Schoenmakers, A.P., \& Roettgering, H.J.A., 2000, MNRAS, 315, 381

Konar, C., Saikia, D.J., Jamrozy, M., \& Machalski J., 2006, MNRAS, 372, 693

Koratkar, A., \& Blaes, O., 1999, PASP, 111, 1

Kunert-Bajraszewska, M., Marecki, A., Thomasson, P., \& Spencer, R.E., 2005, A\&A, 440, 93

Kunert-Bajraszewska, M., Marecki, A., \& Thomasson, P., 2006, A\&A, 450, 945

Lal, D.V., Hardcastle, M.J., \& Kraft, R.P., 2008, MNRAS (in press)

Lasota, J.-P., 2001, New Astronomy Review, 45, 449

Lightman, A.P., \& Eardley, D.M., 1974, ApJ, 187, L1

Lin, D.N.C., \& Shields, G.A., 1986, ApJ, 305, 28 
Marecki, A., Barthel, P.D., Polatidis, A., \& Owsianik I., 2003, PASA, 20, 16

Marecki, A., Thomasson, P., Mack, K.-H., \& Kunert-Bajraszewska, M., 2006, A\&A, 448, 479

Mathur, S., \& Grupe, D., 2005, A\&A, 432, 463

McNamara, B.R., \& Nulsen, P.E.J., 2007, ARA\&A, 45, 117

Merloni, A., \& Nayakshin, S., 2006, MNRAS, 372, 728

Merritt, D., \& Ekers, R.D., 2002, Science, 297, 1310

Meyer, F., \& Meyer-Hofmeister, E., 1981, A\&A, 104, L10

Mineshige, S., \& Shields, G.A., 1990, ApJ, 351, 47

Misra, R., Harikrishnan, K.P., Ambika, G., \& Kembhavi, A.K., 2006, ApJ, 643, 1114

Morganti, R., Tadhunter, C., Oosterloo, T., Holt, J., \& Emonts, B., 2007, ASP Conf. Ser. 373, 343 (arXiv:0710.1197)

Mosoni, L. et al., 2006, A\&A, 445, 413

Murgia, M., Fanti, C., Fanti, R., Gregorini, L., Klein, U., Mack, K.-H., \& Vigotti, M., 1999, A\&A, 345, 769

Nagai, H., Asada, K., Doi, A., Kameno, S., \& Inoue, M., 2008, ASP Conference Series, Vol. 386, p. 290

Narayan, R., \& McClintock, J.E., 2008, New Astr. Rev. 51, 733

Nayakshin, S., Rappaport, S., \& Melia, F., 2000, ApJ, 535, 798

Netzer, H. et al., 1996, MNRAS, 279, 429

Nikolajuk, M., Czerny, B., \& Gurynowicz, P., 2009, astro-ph/0901.1442

O’Dea, C.P., \& Baum, S.A., 1997, AJ, 113, 148

O’Dea, C.P. 1998, PASP, 110, 493

Orienti, M., Dallacasa, D., Stanghellini, C., 2007, A\&A, 461, 923

Osaki, Y, 1974, PASJ, 26, 429 
Owsianik, I., \& Conway, J.E., 1998, A\&A, 337, 690

Owsianik, I., Conway, J.E., \& Polatidis, A.G., 1998, A\&A, 336, L37

Parma, P., Murgia, M., de Ruiter, H. R., Fanti, R., Mack, K.-H., \& Govoni, F., 2007, A\&A, 470,875

Pihlström, Y.M., Conway, J.E., \& Vermeulen, R.C., 2003, A\&A, 404, 871

Polatidis, A.G., \& Conway, J.E., 2003, PASA, 20, 69

Pringle, J.E., Rees, M.J., \& Pacholczyk, A.G., 1973, A\&A, 29, 179

Readhead, A.C.S. et al., 1996, ApJ, 460, 612

Reynolds, C.S., \& Begelman, M.C., 1997, ApJ, 487, L135

Ruszkowski, M., Brueggen, M., \& Begelman, M.C., 2004, ApJ, 611, 158

Sakimoto, P.J., \& Coroniti, F.V., 1981, ApJ, 247, 19

Sanders, J.S., \& Fabian, A.C., 2008, MNRAS, 390, 1

Saripalli, L., et al., 1996, A\&A, 306, 708

Schoenmakers, A.P., de Bruyn, A.G., Roettgering, H.J.A., van der Laan, H., \& Kaiser, C.R., 2000a, MNRAS, 315, 371

Schoenmakers, A.P., de Bruyn, A.G., Roettgering, H.J.A., \& van der Laan, H., 2000b, MNRAS, 315, 395

Shakura, N.I., \& Sunyaev, R.A., 1973, A\&A, 24, 337

Shakura, N.I., \& Sunyaev, R.A., 1976, MNRAS, 175, 613

Siemiginowska, A., \& Czerny, B., 1989, MNRAS, 239, 287

Siemiginowska, A., \& Elvis, M., 1997, ApJ, 482, L9

Siemiginowska, A., Czerny, B., \& Kostyunin, V., 1996, ApJ, 458, 491

Siemiginowska, A., Cheung, C.C., LaMassa, S., Burke, D.J., Aldcroft, T.L., Bechtold, J., Elvis, M., \& Worrall, D.M., 2005, ApJ, 632, 110

Siemiginowska, A., LaMassa, S., Aldcroft, T.L., Bechtold, J., \& Elvis, M., 2008, ApJ, 684, 811 
Sikora, M., Stawarz, Ł., \& Lasota J.-P., 2007, ApJ, 658, 815

Sirothia, S.K., Saikia, D.J., Ishwara-Chandra, C.H., \& Kantharia, N.G., 2008, astro$\mathrm{ph} / 0809.4565$

Smak, J.I., 1982, Acta Astron., 32, 199

Snellen, I.A.G., Schilizzi, R.T., Miley, G.K., de Bruyn, A.G., Bremer, M.N., \& Roettgering, H.J.A., 2000, MNRAS, 319, 445

Snellen, I. A. G., Lehnert, M. D., Bremer, M. N., \& Schilizzi, R. T. 2003, MNRAS, 342, 889

Spergel, D.N., et al., 2003, ApJS, 148, 175

Starling, R.L.C., Siemiginowska, A., Uttley, P., \& Soria, R., 2004, MNRAS, 347, 67

Stanghellini, C., ODea, C.P., Dallacasa, D., Baum, S.A., Fanti, R., \& Fanti, C., 1998, A\&ASS, 131, 303

Stanghellini, C., ODea, C.P., Dallacasa, D., Cassaro, P., Baum, S.A., Fanti, R., \& Fanti, C., 2005, A\&A, 443, 891

Stawarz, Ł., 2004, ApJ, 613, 119

Stawarz, Ł., Ostorero, L., Begelman, M. C., Moderski, R., Kataoka, J., \& Wagner, S., 2008, ApJ, 680, 911

Szuszkiewicz, E., 1990, MNRAS, 244, 377

Taylor, G.B., Marr, J.M., Pearson, T.J., \& Readhead, A.C.S., 2000, ApJ, 541, 112

Tengstrand, O., et al., 2008, A\&A submitted

Tinti, S., \& De Zotti, G., 2006, A\&A, 4889

Tschager, W., Schillizzi, R.T., Roettgering, H.J.A., Snellen, I.A.G., \& Miley, G.K., 2000, A\&A, 360, 887

Turner N.J., 2004, ApJ, 605, L45

van Breugel, W., Miley, G., \& Heckman T., 1984, AJ, 89, 5

Vestergaard, M., \& Peterson, B.M., 2006, ApJ, 641, 689

Vink, J., Snellen, I., Mack, K.-H., \& Schilizzi, R., 2006, MNRAS, 367, 928 
Wilkinson, P.N., Polatidis, A.G., Readhead, A.C.S., Xu W., \& Pearson T.J., 1994, ApJ, 432, L87

This preprint was prepared with the AAS IATEX macros v5.2. 
Table 1. GPS/CSO sample.

\begin{tabular}{|c|c|c|c|c|c|c|c|c|}
\hline name & $z$ & $\begin{array}{c}\text { age } \\
{\left[10^{3} \mathrm{yrs}\right]}\end{array}$ & method & $\begin{array}{r}S_{5 \mathrm{GHz}} \\
{[\mathrm{mJy}]}\end{array}$ & $\begin{array}{c}\log L_{5 \mathrm{GHz}} \\
{\left[\operatorname{erg~\mathrm {s}^{-1}}\right]}\end{array}$ & type & $\log \left(M / M_{\odot}\right)$ & ref. \\
\hline $0035+227$ & 0.096 & 0.567 & kin & 247.0 & 41.41 & $\mathrm{CSO}$ & - & \\
\hline $0108+388$ & 0.668 & 0.417 & kin & 1325.0 & 43.94 & $\mathrm{CSO}$ & - & \\
\hline $0710+439$ & 0.518 & 0.93 & kin & 1629.0 & 43.79 & $\mathrm{CSO}$ & - & \\
\hline $1031+567$ & 0.4597 & 1.8 & kin & 1200.0 & 43.54 & $\mathrm{CSO}$ & - & \\
\hline $1245+676$ & 0.107 & 0.19 & kin & 188.0 & 41.39 & $\mathrm{CSO}$ & - & 5 \\
\hline $1358+624$ & 0.238 & 2.4 & kin & 1838.0 & 43.11 & $\mathrm{CSO}$ & - & 12 \\
\hline $1404+286$ & 0.076 & 0.224 & kin & 2404.0 & 42.18 & $\mathrm{CSO}$ & - & 6 \\
\hline $1843+356$ & 0.763 & 0.18 & kin & 778.0 & 43.83 & $\mathrm{CSO}$ & - & 7 \\
\hline $1943+546$ & 0.263 & 1.306 & kin & 942.0 & 42.91 & $\mathrm{CSO}$ & - & 1 \\
\hline $2021+614$ & 0.227 & 0.368 & kin & 2725.0 & 43.23 & $\mathrm{CSO}$ & - & 8 \\
\hline $2352+495$ & 0.237 & 3.0 & kin & 1538.0 & 43.03 & $\mathrm{CSO}$ & - & 6 \\
\hline B $0840+424$ & 0.35 & 44.0 & kin & 586.0 & 42.97 & $\mathrm{CSO}$ & - & 13 \\
\hline $\mathrm{J} 2203+1007$ & 0.9 & 0.94 & kin & 316.0 & 43.60 & $\mathrm{CSO}$ & - & \\
\hline $\mathrm{J} 1111+1955$ & 0.299 & 1.62 & kin & 642.0 & 42.86 & $\mathrm{CSO}$ & - & \\
\hline $1414+4554$ & 0.186 & 2.03 & kin & 209.0 & 41.94 & $\mathrm{CSO}$ & - & 10 \\
\hline $\mathrm{J} 1415+1320$ & 0.246 & 0.13 & kin & 839.0 & 42.80 & $\mathrm{CSO}$ & - & 10 \\
\hline $\mathrm{J} 1734+0926$ & 0.735 & 1.78 & kin & 909.0 & 43.87 & $\mathrm{CSO}$ & - & 10 \\
\hline $\mathrm{J} 1915+6548$ & 0.486 & 0.94 & kin & 367.0 & 43.08 & $\mathrm{CSO}$ & - & 11 \\
\hline $0221+67$ & 0.31 & 51.0 & syn & 870.0 & 43.03 & GPS/CSS a & - & 14 \\
\hline $0404+76$ & 0.6 & 1.1 & syn & 2820.0 & 44.16 & GPS/CSS a & - & 14 \\
\hline $0740+38$ & 1.06 & 113.2 & syn & 304.0 & 43.73 & GPS/CSS a & 9.51 & 14 \\
\hline $1005+07$ & 0.88 & 18.1 & syn & 2210.0 & 44.42 & GPS/CSS a & - & 14 \\
\hline $1019+22$ & 1.62 & 9.3 & syn & 391.0 & 44.23 & GPS/CSS a & - & 14 \\
\hline $1203+64$ & 0.37 & 35.4 & syn & 1279.0 & 43.36 & GPS/CSS a & - & 14 \\
\hline $1250+56$ & 0.32 & 204.3 & syn & 883.0 & 43.07 & GPS/CSS a & 8.31 & 14 \\
\hline $1323+32$ & 0.37 & 1.4 & syn & 2153.0 & 43.59 & GPS/CSS a & - & 14 \\
\hline $1416+06$ & 1.44 & 50.0 & syn & 1550.0 & 44.72 & GPS/CSS a & - & 14 \\
\hline $1443+77$ & 0.27 & 110.2 & syn & 457.0 & 42.62 & GPS/CSS a & - & 14 \\
\hline $1447+77$ & 1.13 & 151.3 & syn & 457.0 & 43.97 & GPS/CSS a & - & 14 \\
\hline $1607+26$ & 0.47 & 0.5 & syn & 1908.0 & 43.76 & GPS/CSS a & - & 14 \\
\hline $2248+71$ & 1.84 & 26.5 & syn & 306.0 & 44.24 & GPS/CSS a & - & 14 \\
\hline $2252+12$ & 0.54 & 348.2 & syn & 935.0 & 43.59 & GPS/CSS a & - & 14 \\
\hline
\end{tabular}


Table 1-Continued

\begin{tabular}{|c|c|c|c|c|c|c|c|c|}
\hline name & $z$ & $\begin{array}{c}\text { age } \\
{\left[10^{3} \mathrm{yrs}\right]}\end{array}$ & method & $\begin{array}{l}S_{5 \mathrm{GHz}} \\
{[\mathrm{mJy}]}\end{array}$ & $\begin{array}{r}\log L_{5 \mathrm{GHz}} \\
{\left[\operatorname{erg~s}^{-1}\right]}\end{array}$ & type & $\log \left(M / M_{\odot}\right)$ & ref. \\
\hline $2342+82$ & 0.74 & 1.3 & syn & 1280.0 & 44.02 & GPS/CSS a & - & 14 \\
\hline $0810+460 B$ & 0.33 & 10.3 & syn & 266.0 & 42.57 & GPS/CSS a & - & 14 \\
\hline $1025+390 \mathrm{~B}$ & 0.361 & 6.0 & syn & 332.0 & 42.75 & GPS/CSS a? & - & 14 \\
\hline $1233+418$ & 0.25 & 52.7 & syn & 276.0 & 42.33 & GPS/CSS a? & - & 14 \\
\hline $0127+23$ & 1.46 & 0.3 & syn & 1167.0 & 44.61 & GPS/CSS b & - & 14 \\
\hline $0134+32$ & 0.37 & 11.3 & syn & 5727.0 & 44.01 & GPS/CSS b & 8.74 & 14 \\
\hline $0138+13$ & 0.62 & 1.3 & syn & 903.0 & 43.70 & GPS/CSS b & - & 14 \\
\hline $0223+34$ & 1.0 & 0.3 & syn & 1515.0 & 44.38 & GPS/CSS b & - & 14 \\
\hline $0316+16$ & 1.0 & 0.3 & syn & 3032.0 & 44.68 & GPS/CSS b & - & 14 \\
\hline $0428+20$ & 0.22 & 1.2 & syn & 2764.0 & 43.21 & GPS/CSS b & - & 14 \\
\hline $0429+41$ & 1.02 & 0.5 & syn & 4301.0 & 44.85 & GPS/CSS b & - & 14 \\
\hline $0518+16$ & 0.76 & 16.7 & syn & 3652.0 & 44.50 & GPS/CSS b & 8.53 & 14 \\
\hline $0538+49$ & 0.55 & 8.3 & syn & 7637.0 & 44.52 & GPS/CSS b & 9.58 & 14 \\
\hline $0758+14$ & 1.2 & 10.6 & syn & 696.0 & 44.21 & GPS/CSS b & - & 14 \\
\hline $1225+36$ & 1.98 & 0.2 & syn & 812.0 & 44.72 & GPS/CSS b & - & 14 \\
\hline $1328+30$ & 0.85 & 0.3 & syn & 6191.0 & 44.84 & GPS/CSS b & - & 14 \\
\hline $1328+25$ & 1.06 & 1.7 & syn & 3068.0 & 44.74 & GPS/CSS b & - & 14 \\
\hline $1358+62$ & 0.43 & 0.2 & syn & 1838.0 & 43.66 & GPS/CSS b & - & 14 \\
\hline $1458+71$ & 0.9 & 0.6 & syn & 3733.0 & 44.67 & GPS/CSS b & 8.98 & 14 \\
\hline $1517+20$ & 0.75 & 9.6 & syn & 841.0 & 43.85 & GPS/CSS b & - & 14 \\
\hline $1819+39$ & 0.4 & 1.6 & syn & 1179.0 & 43.40 & GPS/CSS b & - & 14 \\
\hline $1829+29$ & 0.6 & 0.8 & syn & 1311.0 & 43.83 & GPS/CSS b & - & 14 \\
\hline $2249+18$ & 1.76 & 0.8 & syn & 822.0 & 44.63 & GPS/CSS b & - & 14 \\
\hline $0345+33$ & 0.24 & 35.8 & syn & 762.0 & 42.73 & GPS/CSS ? & 7.75 & 14 \\
\hline $0809+404$ & 0.55 & 16.1 & syn & 439.0 & 43.27 & GPS/CSS ? & - & 14 \\
\hline $1128+455$ & 0.4 & 1.5 & syn & 659.0 & 43.15 & GPS/CSS ? & - & 14 \\
\hline $1159+395$ & 2.37 & 4.7 & syn & 264.0 & 44.39 & GPS/CSS ? & - & 14 \\
\hline $1225+442$ & 0.22 & 0.8 & syn & 114 & 41.83 & GPS/CSS ? & - & 14 \\
\hline $1242+410$ & 0.811 & 2.6 & syn & 715.0 & 43.85 & GPS/CSS ? & - & 14 \\
\hline $1350+432$ & 2.149 & 1.2 & syn & 28.6 & 43.34 & GPS/CSS ? & - & 14 \\
\hline $\mathrm{J} 0000+4054$ & - & 0.28 & kin & 676.0 & - & CSO? & - & \\
\hline $\mathrm{J} 1826+1831$ & - & 0.38 & kin & 434.0 & - & CSO? & - & 10 \\
\hline
\end{tabular}


Table 1-Continued

\begin{tabular}{ccccccccc}
\hline \hline name & $z$ & $\begin{array}{c}\text { age } \\
{\left[10^{3} \mathrm{yrs}\right]}\end{array}$ & method & $\begin{array}{c}S_{5 \mathrm{GHz}} \\
{[\mathrm{mJy}]}\end{array}$ & $\begin{array}{c}\log L_{5 \mathrm{GHz}} \\
{\left[\mathrm{erg} \mathrm{s}^{-1}\right]}\end{array}$ & type & $\log \left(M / M_{\odot}\right)$ & ref. \\
\hline J0003+4807 & - & 0.34 & kin & 197.0 & - & CSO ? & - & 10 \\
J0204+0903 & - & 0.24 & kin & 715.0 & - & CSO ? & - & 10 \\
J0427+4133 & - & 0.02 & kin & 723.0 & - & CSO ? & - & 10 \\
J0620+2102 & - & 2.06 & kin & 442.0 & - & CSO ? & - & 10 \\
J0754+5324 & - & 2.22 & kin & 286.0 & - & CSO ? & - & 10 \\
J1143+1834 & - & 0.69 & kin & 279.0 & - & CSO ? & - & 10 \\
B0147+400 & - & 6.2 & kin & 254.0 & - & CSO ? & - & 13 \\
$1153+31$ & - & 5.4 & syn & 1.56 & - & GPS/CSS a & - & 14 \\
\hline
\end{tabular}

Note. — References: [1] Polatidis \& Conway (2003); [2] Owsianik et al (1998); [3] Owsianik \& Conway (1998); [4] Taylor et al (2000); [5] Marecki et al (2003); [6] Stanghellini et al (2002); [7] Polatidis (2001); [8] Tschager et al (2000); [9] Polatidis et al (2003); [10] Gugliucci et al (2005); [11] Gugliucci et al (2007); [12] Vink et al (2006); [13] Orienti et al (2007); [14] Murgia et al. (1999). Black hole masses are taken from Woo et al. (2002) and Liu et al. (2006). Different types/classes of sources as explained in Section 2. Ages of the sources with no redshift were estimated assuming $z=0.5$ (Gugliucci et al. 2007). 
Table 2. GPS/CSO sources with given black hole masses.

\begin{tabular}{cccccccc}
\hline \hline name & $\log M / M_{\odot}$ & $\begin{array}{c}\log L_{\mathrm{Edd}}^{a} \\
{\left[\mathrm{erg} \mathrm{s}^{-1}\right]}\end{array}$ & $\begin{array}{c}\log S_{B}^{b} \\
{\left[\mathrm{erg} \mathrm{s}^{-1} \mathrm{~Hz}^{-1}\right]}\end{array}$ & $\begin{array}{c}\log L_{B}^{c} \\
{\left[\mathrm{erg} \mathrm{s}^{-1}\right]}\end{array}$ & $\begin{array}{c}\log L_{\text {bol }}^{d} \\
{\left[\mathrm{erg} \mathrm{s}^{-1}\right]}\end{array}$ & $\dot{m}^{e}$ & $K_{5 G H z}^{f}$ \\
\hline $0740+38$ & 9.51 & 47.62 & 30.76 & 45.72 & 46.72 & 0.12 & 980 \\
$1250+56$ & 8.31 & 46.42 & 29.85 & 44.74 & 45.74 & 0.21 & 470 \\
$0134+32$ & 8.74 & 46.85 & 30.59 & 45.48 & 46.48 & 0.43 & 300 \\
$0518+16$ & 8.53 & 46.64 & 30.88 & 45.82 & 46.82 & 1.49 & 290 \\
$0538+49$ & 9.58 & 47.69 & 30.21 & 45.13 & 46.13 & 0.03 & 41 \\
$1458+71$ & 8.98 & 47.09 & 30.89 & 45.84 & 46.84 & 0.56 & 150 \\
\hline
\end{tabular}

Note. $-{ }^{a}$ Eddington luminosity;

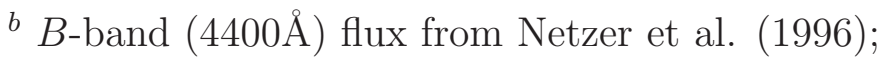

${ }^{c} B$-band $(4400 \AA)$ luminosity;

${ }^{d}$ Bolometric disk luminosity $L_{\mathrm{bol}}=10 \times L_{B}$;

${ }^{e}$ Accretion rate in the Eddington units;

${ }^{f}$ Bolometric correction to $5 \mathrm{GHz}$ monochromatic luminosity. 